(C) 2013 IEEE. Personal use of this material is permitted.

Permission from IEEE must be

obtained for all other uses, in any current or future media, including

reprinting/republishing this material for advertising or promotional purposes, creating new

collective works, for resale or redistribution to servers

or lists, or reuse of any copyrighted

component of this work in other works. 
Randomization" or SPR [4]. However the inter-node

\title{
Free Space Attenuation and Throughput in a Wireless Mobile Network using Successive Interference Cancellation with Power Randomization
}

\author{
Martin J. Tunnicliffe \\ School of Computing and Information Systems, Faculty of Science, Engineering and Computing, \\ Kingston University, Kingston-on-Thames, Surrey, KT1 2EE, UK +20-85472000+62674 \\ e-mail: M.J.Tunnicliffe@kingston.ac.uk
}

\begin{abstract}
Successive Interference Cancellation (SIC) with Power Randomization (SPR) is simulated for a fully meshed wireless network whose nodes may be stationary and equidistant or mobile within a circumscribed area. When the power level differences are just sufficient to allow multi-user detection with equidistant nodes, the introduction of mobility is found to reduce throughput. However when power level differences are dilated, the adverse effect on throughput is not only reduced but reversed when considering group averages over long intervals.
\end{abstract}

Keywords - Multiple Packet Reception, Successive Interference Cancellation, Mobility.

\section{INTRODUCTION}

Successive Interference Cancellation (SIC) allows simultanious transmissions on a common medium to be detected provided (i) their power levels or transmission rates are sufficiently different and (ii) the received power is sufficiently higher than the ambient noise [1]. If transmissions Tx1 and Tx2 have powers $P_{1}$ and $P_{2}$ and rates $R_{1}$ and $R_{2}$ then according to the Shannon Hartley theorem, if Tx2 is treated as interference then Tx 1 can (in principle) be decoded error-free provided $R_{1} \leq B \cdot \log _{2}\left[1+P_{1} /\left(P_{2}+N\right)\right]$ where $N$ is the noise and $B$ is the bandwidth. (This is sometimes called the "packet capture" effect.) If a clean version of Tx1 is subtracted from the received power and $R_{2} \leq B \cdot \log _{2}\left[1+P_{2} / N\right]$, transmission 2 can be decoded as well. This can be applied iteratively to disentangle multiple layers of superimposed signal. The challenge is to choose suitable values of $P$ and $R$ for each transmittier to gain optimum advantage from SIC, maximizing throughput while minimizing power consumption.

SIC has been applied to parallel (MIMO) transmissions to improve spectral efficiency within wireless links [2] and more recently to multi-user random access networks [3,4]. In the absence of centralized control, power is selected randomly from a discrete sequence such that there is (ideally) sufficient difference between any two values to allow multiple detection. This scheme is known as "SIC with Power separations in multi-station environments will be unequal, presenting differing degrees of space-loss, diffraction and fading. In a mobile network these may vary unpredictably, making a nonsense of any precise power-level selection scheme.

Simulations of a fully-meshed network of mobile halfduplex SPR-enabled transceivers have therefore been performed. (We refer to the network as "fully meshed" since all nodes are theoretically capable of sending to all others; there is no forwarding of packets between nodes as in an ad hoc network.) The nodes generate packets according to a Bernoulli process with optional media access control (MAC) mechanism whereby random backoffs are initiated if outgoing and incoming packets coincide. It does not represent any real wireless protocol but rather a generic paradigm. Data rate is assumed constant for all nodes, while power levels are selected randomly from a discrete sequence in an identical manner to that described in [4]. The experiment was designed to identify and find ways to ameliorate any adverse effects mobility (and subsequent power randomization) might have on SPR efficiency and throughput.

\section{Simulation APPROACH}

The simulator was written in $\mathrm{C}++$ and represents an arbitrary number of mobile nodes moving across a plane disk. The nodes pass messages to each other via proxy objects which model the free-space attenuation between them. (The effects of fading were not included in the current study.) All physical units are normalized: powers are multiples of the ambient noise and data rates are multiples of the analogue bandwidth such that the Shannon-Hartley theorem simplifies to $R=\log _{2}(1+P)$. Distances are specified as multiples of the length over which the smallest transmitted power attenuates to the noise level. Antennas are assumed to be omnidirectional within the plane of the disk. Time is measured in discrete "steps", each representing the period required to process one unit of data at unity rate. 


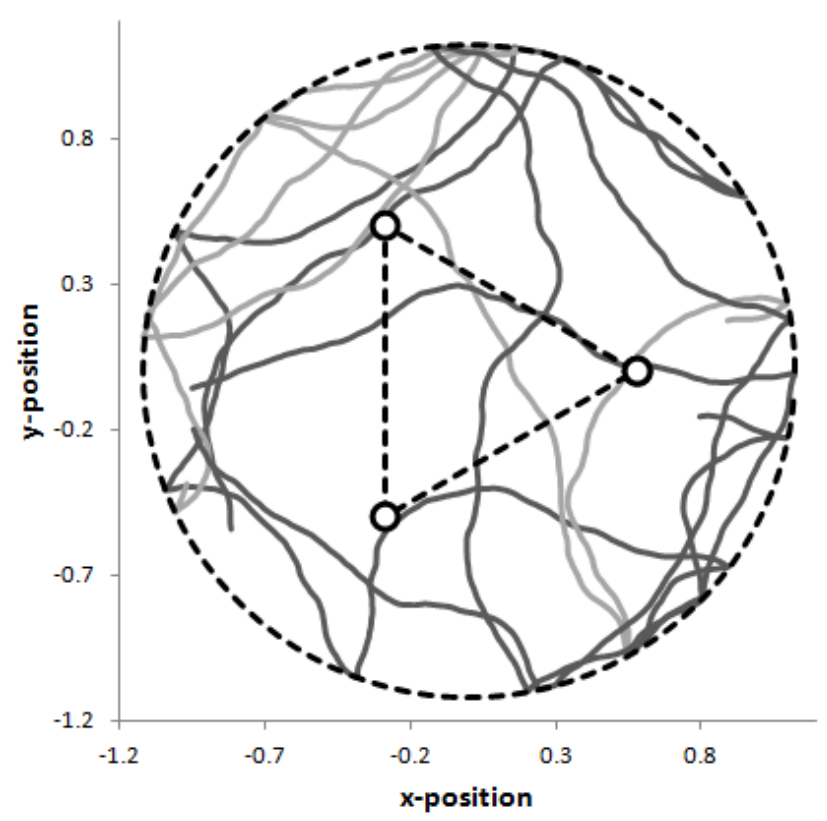

Figure 1. Typical trajectories of a three-node mobile network with $\operatorname{Med}(l)=1 \quad(r=1.122)$. The equivalent three-node stationary network $(l=1)$ is also shown.

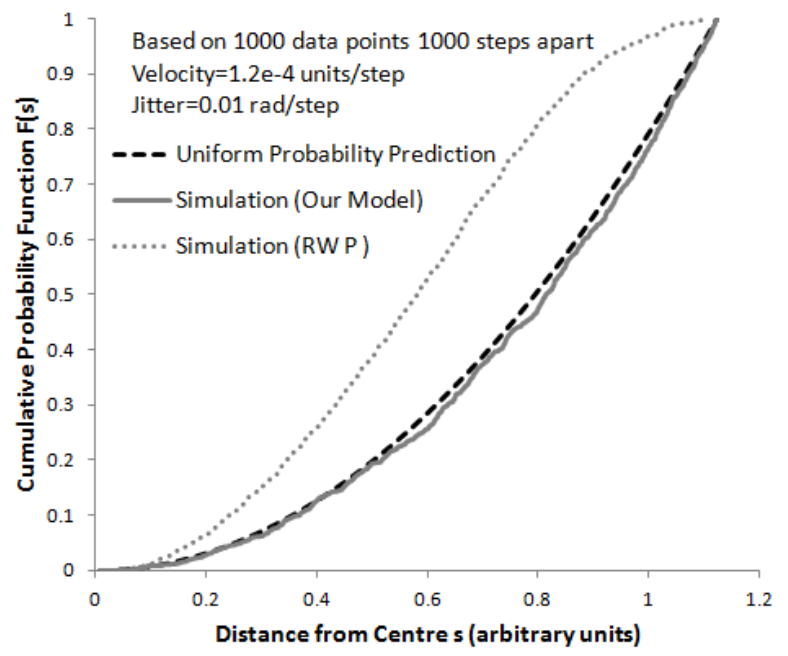

Figure 2. Cumulative probability function compared with the uniform areal node distribution $\left(F(s)=(s / r)^{1 / 2}\right)$. RWP results are shown for comparison.

\section{NODE MOBILITY}

Mobility is frequently modelled using the Random Waypoint Model (RWP) in which nodes move at uniform speed between randomly selected waypoints. However this tends to produce a nonuniform distribution; the highest probabilities are clustered around the system centre while the borders are underrepresented [5]. An alternative approach was therefore used whereby each node moves a fixed distance $(0.0001$ of the disk radius) per time step in a direction which is subjected to random Gaussian perturbations. If during a time step a node attempts to leave the system, its direction is randomized and that step is repeated. (Figure 1 shows some typical node trajectories.) The resulting distribution is almost uniform - a fact which can be demonstrated by comparing the cumulative probability function obtained from 1,000,000 steps with the expected distribution for a uniform distribution, i.e. $F(s)=\sqrt{s / r}$ where $s$ is the distance from the system centre and $r$ is the system radius. Figure 2 shows the two distributions to be almost identical, while the RWP distribution is signigficantly different. The uniform density allows easy computation of the median transmission length, allowing stationary and mobile networks to be compared like-with-like. Assuming a uniform distribution, the distance $l$ between nodes follows the p.d.f. $f(l)=\frac{4 l}{\pi r^{2}}\left[\arccos \frac{l}{2 r}-\frac{l}{2 r} \sqrt{1-\frac{l^{2}}{4 r_{2}}}\right]$ [5] whose median can numerically be shown to be $0.89129 r$. Thus the system radius corresponding to a unity median is $r=1.122$. The largest static network in which all nodes are equal distance from their neighbours is the equilateral triangle shown on Figure 1, so this geometry was used to compare performance of static and mobile networks with equal median node separations. (Theoretically a 4-node network could be represented by a regular tetrahedron, but this would require three dimensions.)

\section{TRANSMITTER FUNCTIONALITY}

All data messages are 1000 units long and are transmitted at unity rate such that each occupies 1000 time steps. Each node is assigned a mean message interarrival time such that an interarrival time of 1000 constitutes 1 Erlang of traffic. Individual arrivals are generated by a Bernoulli process, the per-step arrival probability being the reciprocal of the mean interarrival time. Across the entire 1,000,000 step simulation this can be considered to be approximately Poissonian. All nodes in the network have identical parameters, though their behaviours are statistically independent. The offered traffic can be varied between 0 and 1 Erlang by increasing the average number of arrivals per 1,000,000 slots between 0 and 1000 in steps of 10. For each experiment the throughput (the successfully received traffic in Erlangs) is recorded.

The nodes can work in both broadcast and unicast modes: in broadcast mode each node transmits messages to all its neighbours but without any MAC functionality; data is sent as soon as it is ready without regard for 


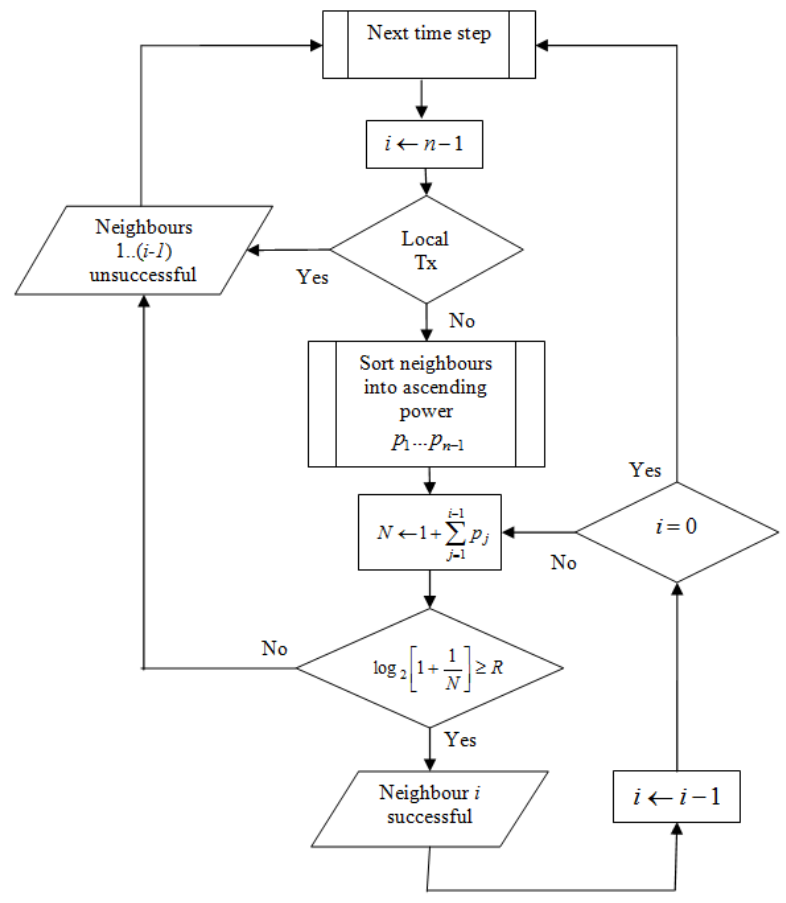

Figure 3. Simulated decoding of multiple packets from $N$ neighbours. Silent nodes are classified as producing zero power.

whoever else might be transmitting. In unicast mode however, each message has a randomly selected recipient and a primitive MAC is implemented: if at the instant a message is ready for transmission an incoming message is being received, the new message is buffered and backs off for a random period between 1 and 1000 time steps before re-trying. A minimum interval between successive messages is also enforced, equal to 1000 steps plus an additional random number of steps between 1 and 1000 .

\section{POWER LEVEL SELECTION}

The random selection of power levels to optimize the capture effect has been discussed since at least the late 1980's [6]. More recent workers have considered selection from discrete power sequences to optimize the recovery of the weaker signal also [7] and $\mathrm{Xu}$ et al. [8] recently proved that discrete sequences work best under realistic coding algorithms. Here we follow [1] and [4] in assuming ideal coding, e.g. error-free detection right up to the Shannon limit. A message received with power $P$ and rate $R$ can be conveniently characterised with figure-of-merit $\alpha=P /\left(2^{R}-1\right)$ which increases with increasing power - at the expense of battery lifetime and with reducing $R$ - at the expense of transmission speed. (You are more likely to be understood if you speak louder and/or more slowly.) If the inter-node attenuations are equal then all concurrent transmissions should be simultaneously decodable if the unique values $\left\{\alpha_{1}, \alpha_{2} \ldots\right\}$ assigned to different senders obey the inequality $\alpha_{i} \geq \sum_{j=1 . i-1} P_{j}+1, \forall i>0$. If we assume a constant transmission rate $R=1$ then the power sequence

$$
P_{i}=\left(2^{R}-1\right) \cdot\left[\sum_{j=0}^{i-1} P_{j}+1\right], \quad P_{0}=0
$$

ensures that unless the receiving node is sending all unequal power transmissions should be simultaneously decodable.

\section{RECEIVER FUNCTIONALITY}

Figure 3 shows the algorithm used to simulate receiver functionality. All incoming transmissions are discarded if the local transmitter is sending, otherwise they are ranked in descending power. If the strongest signal is distinguishable from the combined power of all weaker signals and noise, it is deemed successful and the process is repeated for the next strongest. Unsuccessful signals are discarded along with all weaker transmissions. Note that in unicast mode transmissions are often promiscuously decoded to extract the desired signal; in a real system detection may be achieved with the linear minimum mean square error (MMSE) technique [2] but no attempt is made here to model the specifics of decoding.

\section{FREE SPACE ATtENUATION}

Free-space attenuation between nodes is modelled by a simple inverse-square relation, the received power $P(l)=P_{1} / l^{n} \quad$ where $l$ is the distance between transmitter and receiver, $P_{1}$ is the received power when $l=1$, and $n$ is the free space path loss exponent. We assume $n=2$ for ideal spherical wavefronts, though in practice $n>2$ (and even $n<2$ ) are typically needed to model practical scenarios with diffraction and specular reflections.

\section{POWER DILATION}

The relative movement of the nodes causes power levels to vary greatly from the values required by Eqn.(3) with an inevitable effect on performance. To compensate for this, a "dilation" factor $k \geq 1$ is used to add some slack into the system; this reduces the 


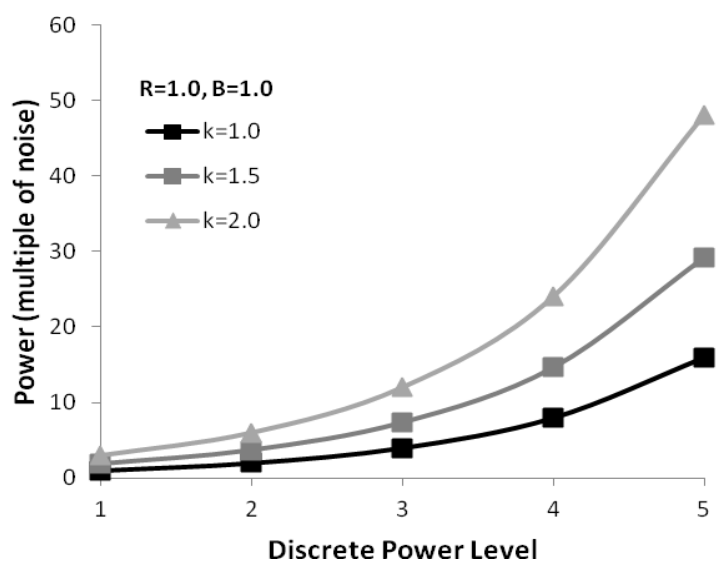

Figure 4. Power level sequences for three values of dilation factor $k$.

analogue bandwidth assumed in the power level calculation such that

Figure 4 shows the first five power levels plotted against $k$. Note that an increase in $k$ from 1 to 2 means more than doubling of the power level. This may impose limits on the range of dilation in mobile nodes which have limited power resources.

\section{RESULTS}

Figures 5 and 6 show the effect of increasing dilation from 1.0 to 2.0 on the throughput of stationary and mobile 3-node networks with three random power levels at different intensities of offered traffic. The horizontal and vertical axes represent the offered and carried traffic per transmitter per receiving neighbour respectively. Figure 5 represents a broadcast network with the MAC mechanism disabled, in which the throughput falls to zero as the offered traffic approaches 1 Erlang (at which point all three stations are nearly always transmitting

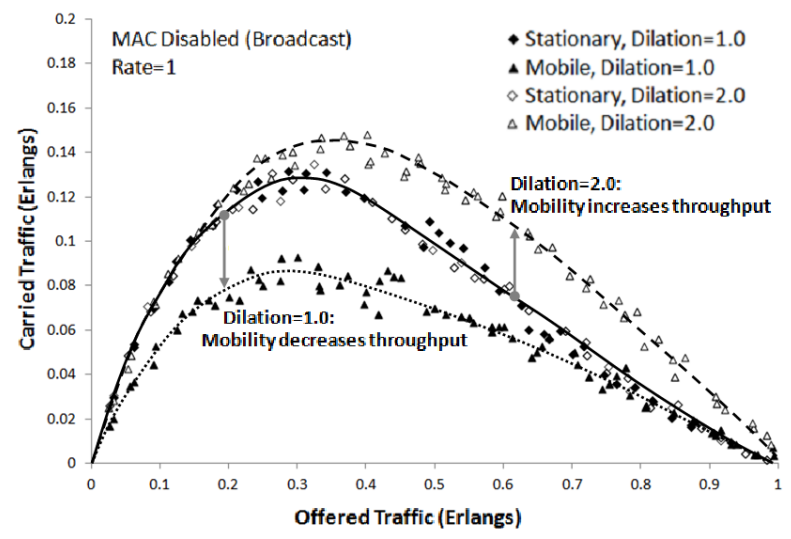

Figure 5. Effect of mobility on throughput for a 3-node network with and without power-level dilation with MAC disabled. and are therefore unable to receive). Without mobility, dilation has no noticable effect upon throughput. When $k=1.0$ (no dilation) the introduction of mobility significantly reduces throughput except when the traffic is extremely high. Figure 6 shows the maximum observed throughput as a function of the dilation factor between 1 and 2, showing the point at which the mobility starts to become beneficial. This occurs around $k=1.5$ when MAC is disabled (broadcast), and about $k$ $=1.35$ when MAC is enabled (unicast). Figure 7 shows the optimum throughput for larger networks (keeping the number of levels to 3) showing that dilation continues to improve throughput in networks of 8nodes.

If $k$ is increased to 2.0 a surprising result is seen: for

$$
P_{i}=\left(2^{k R}-1\right) \cdot\left[\sum_{j=0}^{i-1} P_{j}+1\right], \quad P_{0}=0 \text {. }
$$

utilizations $<0.2$ Erlangs the mobility has no noticeable effect upon throughput while under higher traffic the mobility increases throughput. A similar phenomenon is seen in Figure 6, which was obtained using a unicast 3node network. Here the MAC mechanism regulates medium access, preventing the near-zero throughput under high traffic seen in Figure 4. Again dilation has no effect on throughput when the nodes are static and equidistant, but when $k=2.0$, mobility has a beneficial effect (except when offered traffic is below 0.1 Erlang, in which case there is no significant change).

Figure 7 shows the maximum observed throughput as a function of the dilation factor between 1 and 2, showing the point at which the mobility starts to become beneficial. This occurs around $k=1.5$ when MAC is disabled (broadcast), and about $k=1.35$ when MAC is enabled (unicast). Figure 8 shows the optimum throughput for larger networks (keeping the number of

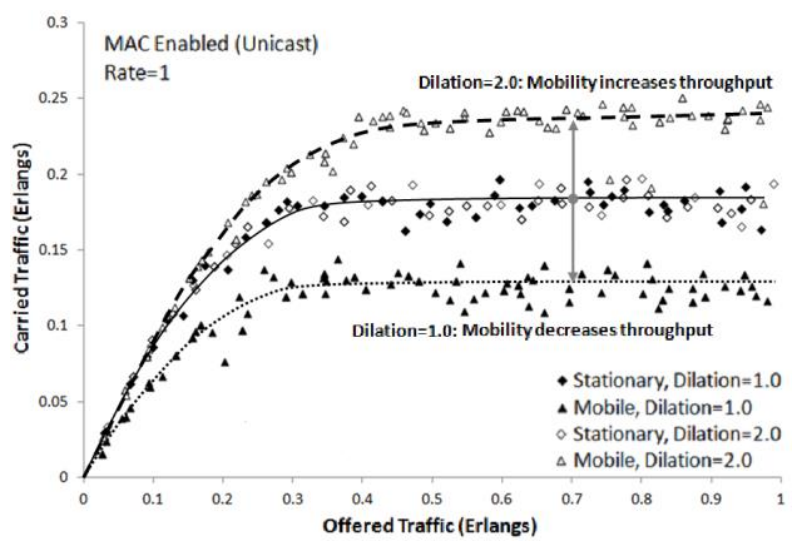

Figure 6. Effect of mobility on throughput for a 3-node network with and without power-level dilation with MAC enabled 


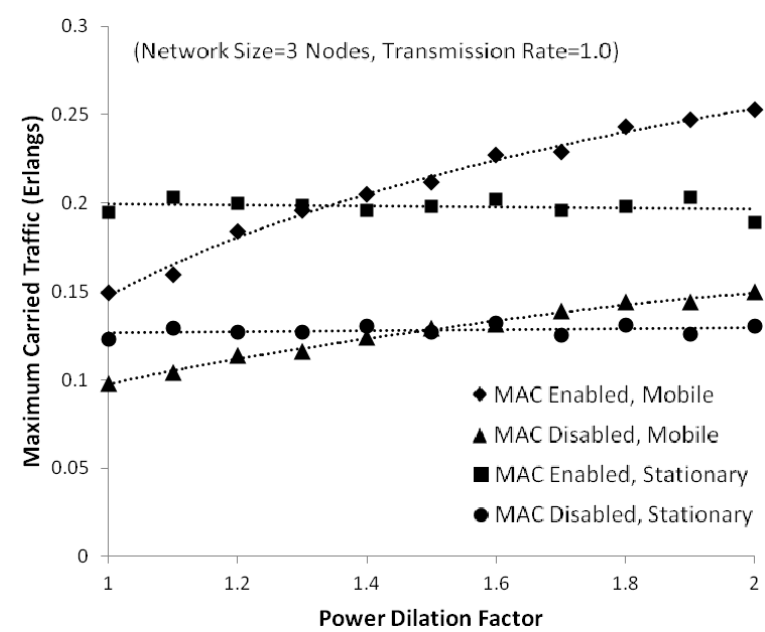

Figure 7. Maximum throughput vs. dilation factor with and without mobility.

power levels to 3) showing that dilation continues to improve throughput in networks of 8 mobile nodes.

\section{DisCuSSION AND CONCLUSIONS}

The reported investigations demonstrate the performance of SIC with Power Randomization in a network of wireless nodes. While other workers have addressed SIC in wireless environments [10], here we include the power randomization effect introduced by mobility. The physical environment is assumed to be ideal (although some other workers have considered multiple packet reception under Rayleigh fading [9].) Furthermore receivers are assumed to be able to decode error-free up to the ideal rate. Furthermore the media access mechanism is a generic implementation of CSMA/CA which does not represent any real wireless LAN protocol. Under these conditions, when the differences between successive power levels were just sufficient to allow multiple signal detection between equidistant nodes, the introduction of mobility was found to reduce throughput, while when the power level differences were dilated the adverse effect of mobility on throughput was not only reduced but reversed. (Power-level dilation had no effect on throughput when nodes were stationary and equidistant.)

It should be stressed that only relatively simple scenarios have yet been tested. All the nodes in the network are assumed to have the same behavioral statistics, moving with the same statistical characteristics and transmitting at the same average rate. In addition, the results refer to the group mean of the entire network averaged over long periods of consistent behavior. The perceived benefits and drawbacks for single nodes over shorter time periods have yet to be investigated.

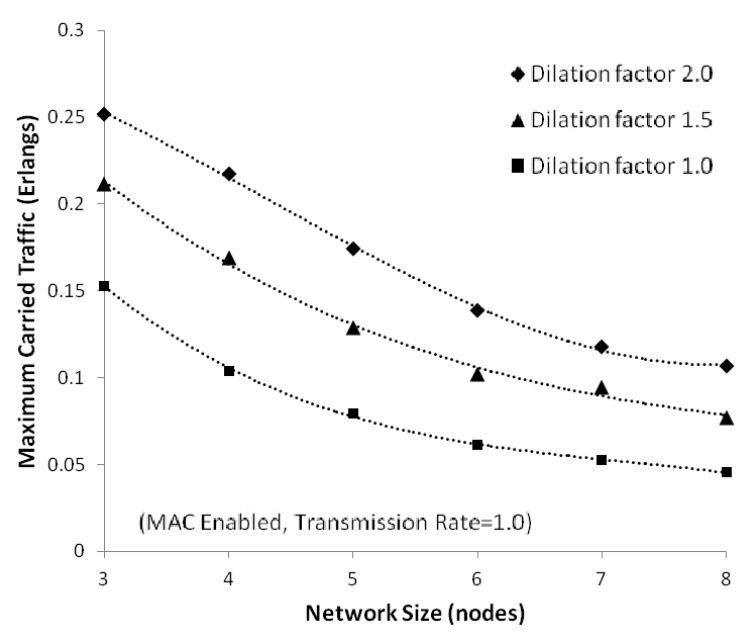

Figure 8. Max. throughput vs.network size for MAC-enabled unicast network.

\section{ACKNOWLEDGMENT}

The author thanks colleagues at Kingston and City University Hong Kong for support and encouragement.

\section{REFERENCES}

[1] S. Sen, N. Santhapuri, R.R. Choudhury, S. Nelakuditi, "Successive Interference Cancellation: A Back-of-the-Envelope Perspective", in Proc. 9th. ACM SIGCOMM Workshop on Hot Topics in Networks (Hotnets '10), Monetray, CA, 20-21 Oct. 2010, pp.1-6.

[2] A. Zanella,A, M. Chiani, M.Z. Win, "MMSE Reception and Successive Interference Cancellation for MIMO Systems with High Spectral Efficiency", IEEE Trans. Wireless Comm., 4(3), pp.1244-53, 2005.

[3] D.Halperin, T.Anderson, Wetherall,D, "Taking the Sting out of Carrier Sense: Interference Cancellation for Wireless LANs", in Proc. 14th ACM International Conference on Mobile Computing and Networking, San Francisco, 14-19 Sept. 2008, pp.339-50.

[4] M. Zou, S. Chan, H.L. Vu, V. Xu, L. Ping, "Optimal Throughput for 802.11 DCF with Multiple Packet Reception", Proc. 36th Annual IEEE Conference on Local Computer Networks, Bonn, Germany, 4-7 Oct. 2011, pp.601-7.

[5] Bedstetter,C, Hartenstein,H, Peréz-Costa,X, "Stochastic Properties of the Random Waypoint Mobility Model", Wireless Networks, 1(5), pp.555-67, 2004.

[6] R.O. LaMaire, A. Krishna, M. Zorzi, "On the Randomization of Transmitter Power Levels to Increase Throughput in Multiple Access Radio Systems", Wireless Networks, 4, pp.263-77, 1998. 
[7] Zanella,A, Zorzi,M, "Theoretical Analysis of the Capture Probability in Wireless Systems with Multiple Packet Reception Probabilities", IEEE Trans. On Communications, 60(4), pp.1058-71, 2012.

[8] C. Xu, P. Wang,P, S. Chan, L. Ping, "Decentralised Power Control for Random Access with Multi-User Detection, Proc. $7^{\text {th }}$ Int. Symp. On Turbo Codes and Interactive Processing, Gothenburg, Sweden, 27-31 July 2012, pp.1-11, 2012.

[9] A. Dua, "Random Access with Multiple Packet Reception", IEEE Trans. Wireless Communication, 7(6), pp.2280-8, 2008.

[10] S.P. Weber, J.G. Andrews, X Yang, G de Veciana, "Transmission Capacity of Wireless Networks with Successive Interference Cancellation", IEEE Trans. On Information Theory, 53(8), pp.2799-814, 2007. 